\title{
Driver Lane Change Intention Inference for Intelligent Vehicles: Framework, Survey, and Challenges
}

\author{
Yang Xing, Chen Lv, Member, IEEE, Huaji Wang, Hong Wang, Yunfeng Ai, Dongpu Cao, Member, \\ IEEE, Efstathios Velenis, Fei-Yue Wang, Fellow, IEEE
}

\begin{abstract}
Intelligent vehicles and advanced driver assistance systems (ADAS) need to have proper awareness of the traffic context as well as the driver status since ADAS share the vehicle control authorities with the human driver. This study provides an overview of the ego-vehicle driver intention inference (DII), which mainly focus on the lane change intention on highways. First, a human intention mechanism is discussed in the beginning to gain an overall understanding of the driver intention. Next, the ego-vehicle driver intention is classified into different categories based on various criteria. A complete DII system can be separated into different modules, which consists of traffic context awareness, driver states monitoring, and the vehicle dynamic measurement module. The relationship between these modules and the corresponding impacts on the DII are analyzed. Then, the lane change intention inference (LCII) system is reviewed from the perspective of input signals, algorithms, and evaluation. Finally, future concerns and emerging trends in this area are highlighted.
\end{abstract}

Index Terms - Intelligent vehicle, ADAS, lane change, driver intention, parallel driving.

Copyright (c) 2015 IEEE. Personal use of this material is permitted. However, permission to use this material for any other purposes must be obtained from the IEEE by sending a request to pubs-permissions@ieee.org.

The work is supported by National Natural Science Foundation of China (61533019, 91720000) and Beijing Municipal Science \& Technology Commission (Z181100008918007). Also supported in part by the Young Elite Scientist Sponsorship Program by CAST (No. 2017QNRC001) and the SUG-NAP Grant (No. M4082268.050) of Nanyang Technological University, Singapore. (Corresponding Authors: Dongpu Cao, Hong Wang)

Y. Xing is with the Advanced Vehicle Engineering Centre, Cranfield University, Bedford MK43 0AL, U.K., and with Vehicle Intelligence Pioneers Ltd, Qingdao, 266000, China (e-mail: y.xing@,cranfield.ac.uk).

C. Lv, is with the School of Mechanical and Aerospace Engineering, Nanyang Technological University, 639798, Singapore. (e-mail: lyuchen@ntu.edu.sg).

H. J. Wang is with the AVL Powertrain UK Ltd, Coventry, CV4 7EZ, UK. (e-mail: Huaji.wang@avl.com).

E. Velenis is with the Advanced Vehicle Engineering Centre, Cranfield University, Bedford, MK43 0AL, UK (e-mail: e.velenis@cranfield.ac.uk).

H. Wang and D. P. Cao are with Mechanical and Mechatronics Engineering with the University of Waterloo, 200 University Avenue West Waterloo, ON, N2L3G1, Canada (e-mail: hong.wang, dongpu.cao@uwaterloo.ca).

Y. F. Ai is with the School of Artificial Intelligence, University of Chinese Academy of Sciences, Beijing 100049, China (e-mail: aiyunfeng@ucas.ac.cn)

F. Y. Wang is with the State Key Laboratory of Management and Control for Complex Systems, Institute of Automation, Chinese Academy of Sciences, Beijing 100190, China (e-mail: feiyue@ieee.org).

\section{INTRODUCTION}

$\mathrm{M}$ ore than $80 \%$ of traffic accidents were caused by driver errors [1]-[3]. Until now, various passive safety systems like airbags and seat-belts have played a significant role in the protection of the driver and passengers. Although these techniques have saved millions of lives, they are not designed to prevent accidents from happening but to protect the passengers after the accidents [4] [5]. Instead of minimizing the injuries after the accidents, many efforts have been devoted to the development of safer and more intelligent systems such as the ADAS techniques so that the accidents can be prevent from happening. ADAS techniques like Adaptive Cruise Control (ACC), lane departure avoidance (LDA), lane keeping assistance (LKA), and side warning assistance (SWA) can assist the driver in making right decisions and reducing their workloads [6]-[8]. However, these systems usually make decisions without taking driver intended maneuver into consideration. A driver is in the center of the Traffic-Driver-Vehicle (TDV) loop, who makes decisions and interact with other road users by controlling the vehicle. Hence, understanding driver intention and behaviors are beneficial to driver safety, vehicle drivability, and traffic efficient.

From the cognitive psychology perspective of view, intention refers to the thoughts that one has before the actions [9]. Accordingly, driver intention is the attitude towards performing a series of future vehicle control actions. Three aspects determine the human intention: the attitude towards the behavior, subjective norm and the perceived behavior control [10]. Bratman defined the intention as the main attitude that directly influences future actions [11]. Also, Heinze described a triple level architecture of the intentional behavior, which consists of intended level, activity level, and state level [12].

Within the human-machine-interaction (HMI) scope [13], intention refers to the thoughts or attitudes towards an on-going action. Accordingly, intention recognition is the process of understanding whether the on-going activities of the agent are goal-oriented or not, and what is the goal behind these specific actions. Bonchek and Elisheva proposed a cognitive model with two core components, which were intention detection and intention prediction [14]. Intention detection is a process of analyzing whether a sequence of actions has underlying intention. Intention prediction, on the other hand, refers to the 
prediction of the intentional goal based on a set of incomplete sequence of actions. The intention inference and reasoning make people intelligent and enable them to be effectively involved in interpersonal activities and communications. A human can recognize other's intention based on their observation and the learned social knowledge. From the HMI perspective of view, only when a robot can recognize human intention based on their own observations can they be viewed as an intelligent agent [15]-[23]. Regarding the intelligent vehicles, it is still difficult to learn how to infer human intention accurately and collaborate with the driver efficiently since most of the most of current intelligent vehicles lack the ability of self-learning and knowledge summarizing by themselves.

The reasons for developing the DII technique are multi-folds. One of the primary motivations is to improve driving safety. Inferring the driver intention can better assess the potential risks. Since a large amount of accidents are caused by human errors, misbehavior, cognitive and judgment errors [24]-[26], monitoring and correcting the driver intention in time are critical to the ADAS. Also, intention recognition enables the ADAS to avoid making conflict decisions with the driver [27]. As ADAS share the control authorities with the driver, it is essential for the ADAS to recognize the driver intention and not operate against the driver's will. Driver intention inference enables the ADAS to assist the driver and focus on the corresponding traffic context perception as early as possible.

Furthermore, intention inference system will contribute to the development of automated vehicles. DII system can be used to modeling the driver intention and generate human-like decision-making system. Concerning the level-three automated vehicles (SAE international standard, J3016), accurate driver intention prediction will contribute to a smoother and safer transition between the driver and the autonomous vehicle controller [28]-[30]. When level-three automated vehicle working in the automated driving mode, all the driving tasks are handled by the vehicle. However, once an emergent situation occurs, it must disengage and give the driving authority back to the driver. The vehicle can determine whether the driver is ready to take over or not by assessing their intention in advance.

The contribution of this study can be summarized as follow. First, a state-of-art literature review for driver lane change intention is proposed. The LCII system is categorized based on different criteria. Second, the critical time flow of the DII with different driver behaviors is introduced. This leads to a comprehensive understanding of the architecture of intention inference system. Finally, future works and challenges of DII are proposed, and a parallel driver intention inference system is introduced.

This paper is organized as follow. In Section II, general human intention mechanisms and the classification methods for the DII systems are reviewed. In Section III, the time-flow of the LCII system is introduced, and literature on LCII are reviewed from different perspective of views. In Section IV, future works and research are discussed. Finally, the paper is concluded in Section V.

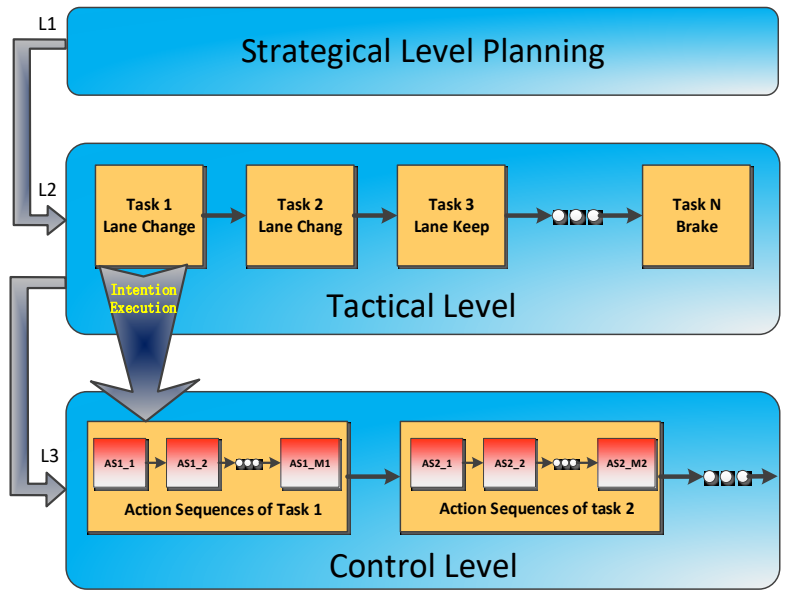

Fig. 1. Driver intention classification based on the time constant.

\section{DRIVER INTENTION CLASSIFICATION}

Driver intention can be classified into different categories based on different criteria. For example, it can be classified according to the motivation, time-scale, and the vehicle control direction. Among these, the two most straightforward classification ways are based on the time-scale of the intention and the driving directions.

\section{A. Time-Scale based Driver Intention Classification}

Michon stated that the cognitive structure of human behavior in the traffic environment is a four-level hierarchical structure, which consists of road user, transportation consumer, social agent, and psycho-biological organism [31]. Among these, the road user level is directly related to the drivers and can be further divided into three sub-levels: strategical, tactical, and operational level (also known as control level), respectively, as shown in Fig. 1. The three cognitive levels can be viewed as three intentional levels based on time-scale characteristics. Strategy level defines the general plan of a trip such as the trip route, destination, and risk assessment, etc. The time constant is at least in minutes or even longer. At this moment, the driver considers transport mobility and comfortable issues, which is a long time-scale problem. Regarding the tactical level, the driver will make a short-term decision and control the vehicle to negotiate the prevailing circumstance. Tactical intentional maneuvers can consist of a series of operational maneuvers to finish the short-term tactical goals such as the turning, lane changing, and braking maneuvers [32].

The control commands must meet the strategy that are defined in the strategical level. The control intention is the shortest maneuver among the three and stands for the will to remain safe and comfortable. The time constant of the control action is generally in milliseconds. Also, Salvucci et al. concluded that lane change was not merely a control procedure, but also incorporated a set of critical aspects of driving such as lower level controls [33]. For example, lane change maneuver can contain a series of short-term driving behaviors like the acceleration and deceleration in the longitudinal direction and the steering wheel control in the lateral direction. 


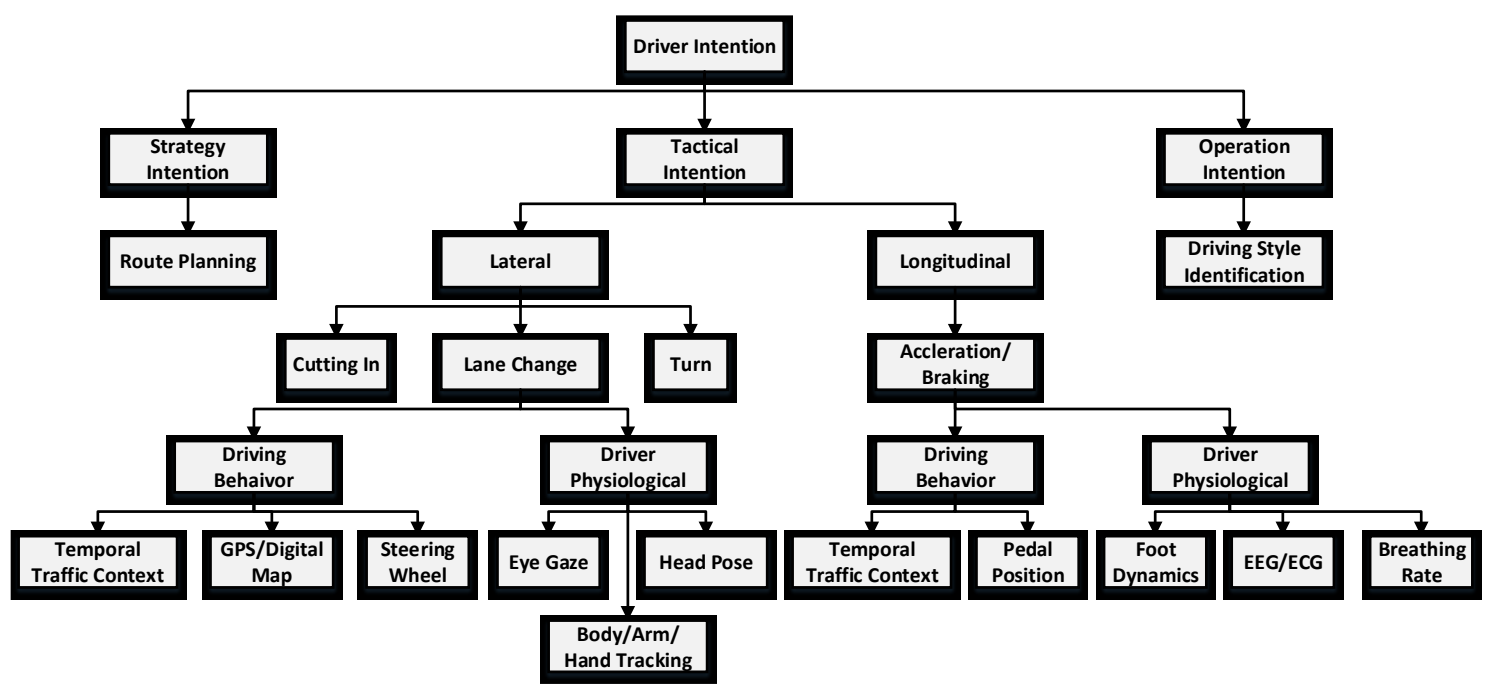

Fig. 2. Taxonomy of driver intention systems.

A driver model, namely, Adaptive Control of Thought-Rational cognitive architecture was developed in [34]. Like the three-level architecture given by [31], the integrated driver model is divided into another three components, which are control, monitoring, and decision-making module. The control component is similar to the control level in [31], which is responsible for the perception of the external world and transfer the control signals to the vehicle. The monitoring component keeps aware of the surrounding situation and context by periodically perceiving and analyzing. The decision-making component, which has the same function with Michon's tactical level, makes tactical decisions for each maneuver according to the awareness of the current situation and the information gathered from the control and monitoring module. One significant advantage of the cognitive driver model is the incorporation of the built-in features are useful for human ability imitation. A taxonomy of driver intention classification is depicted in Fig. 2

\section{B. Directional-based Driver Intention Classification}

The longitudinal and lateral motion are two basic directions for underground vehicles. Driver's longitudinal behaviors contain braking, acceleration, starting, and lane keeping, etc. While the lateral intentions are normally more complicated than the longitudinal intention dues to the complex interaction with surrounding vehicles.

Regarding the longitudinal intention, most of the previous studies focus on braking intention recognition [35]. Haufe et al. proposed a driver braking intention prediction using EEG (electroencephalograph) and EMG (electromyography) signals [36]. Similarly, Khaliliardali et al. proposed a driver intention prediction model to determine whether the driver will go ahead or stop based on the brain-computer-interface (BCI) technique [37]. McCall and Trivedi integrated the DII into an intelligent braking assistance system [38]. A sparse Bayesian learning algorithm was used to infer the driver's braking intention. Trivedi et al. predicted the driver's braking intention by directly monitoring the foot gesture through cameras [39] [40]. They showed that the driver foot gesture estimation plays a vital role in the vehicle longitudinal control and the usage of vision-based foot tracking is more straightforward and accurate. [41] [43] provided the braking intention estimation methods at intersections. Takahashi et al. predicted the deceleration intent during downhill road [42].

In addition, tactical maneuvers usually consist of a series of sub-control maneuvers. Some of the existing studies focus on the analysis of multiple tactics rather than a single tactical task based on the utilization of machine learning methods. It was mentioned that discriminative machine learning models are more suitable for binary intention classification, while generative methods contribute to a higher intention detection accuracy for the multi-intention inference tasks [44]-[47].

\section{DRIVER INTENTION INFERENCE METHODOLOGIES}

\section{A. Architecture of Driver Intention Inference System}

DII system is an integration of multiple techniques such as perception, data fusion and synchronization, model learning and model inference. According to the existing studies, DII system mainly contains the following modules: traffic context perception module, vehicle dynamic module, driver behavior recognition module, and driver intention inference module.

In Fig. 3, the traffic context information is captured by the environment perception block. This block captures the road and traffic context and outputs the position information of the ego-vehicle. By integrating the environment perception module with vehicle dynamic data through the CAN bus or the Ethernet, the relative distance, velocity, and future motion of the ego-vehicle and the surrounding vehicles can be obtained. The traffic and vehicle dynamic data will be fed into the inference module along with the driver behavior information. The driver behavioral information usually contains driver head rotation, eye gaze, and body movement, etc. Next, the intention inference model will calculate the probability of a lane change intention based on the integrated information. Like the human driver, the final output of the lane change decision module is a binary value which indicates a specific lane change decision. After the lane change decision is activated, the interaction module models the driver hand and foot dynamics as well as the dynamics of vehicle control interface. 


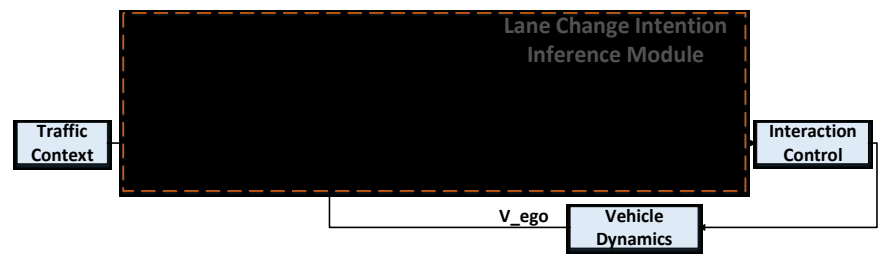

Fig. 3. Driver lane change intention inference framework.

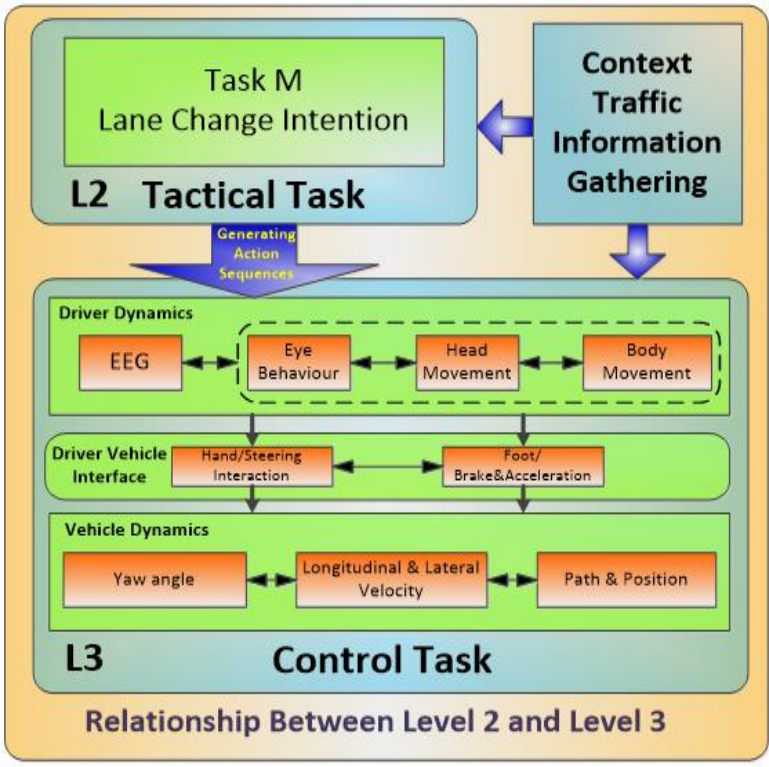

Fig. 4. Relationship between tactical intention and control intention.

TABLE 1

COMMON INPUT SigNALS AND SENSORS USED FOR DRIVER INTENTION INFERENCE.

\begin{tabular}{ll}
\hline \hline Sensor Sources & Sensor Categories \\
\hline Traffic & $\begin{array}{l}\text { Current ego-vehicle position (collected with GPS and } \\
\text { digital map), Relative distance, velocity and acceleration } \\
\text { concerning the front and surrounding vehicles (collected } \\
\text { with cameras, radar or Lidar). }\end{array}$ \\
\hline Vehicle & $\begin{array}{l}\text { CAN bus signals (including steering wheel angle, steering } \\
\text { wheel velocity, brake/gas pedal position, velocity, heading } \\
\text { angle, etc.) }\end{array}$ \\
\hline Driver & Cameras (Head rotation, gaze direction, foot dynamics). \\
& EEG, EMG, Heart rate, etc. \\
\hline \hline
\end{tabular}

The relationship between the tactical intention and the control intention concerning the driver-vehicle-interaction is explicitly illustrated in Fig. 4. Fig. 4 contains three parts namely the traffic context perception, tactical intention unit, and control units. Specifically, in the third level, three layers are defined. The upper layer is driver dynamics, which represent the checking and monitoring behavior of the driver. In this part, the most common dynamics are the brain dynamics that are measured by the EEG device, eye gaze behavior, head movement, and body movement (hand, body, and foot dynamics, etc.). The driver-vehicle-interaction layer will be activated once the lane change decision is made. Finally, vehicle control signals are fed into the lowest vehicle control layer.

\section{B. Inputs for Driver Intention Inference System}

The driver is in the center of the TDV loop. The signals from the TDV loop that used for driver intention inference can be classified into three categories. The standard inputs for the DLII system are summarized in Table 1.

\section{1) Traffic Context}

Traffic context is the primary stimuli to the driver intention. A better understanding of the surrounding traffic context will improve the intention inference accuracy. For instance, a lane change maneuver usually occurs when encountering a low speed front vehicle or a rear vehicle is approaching with fast speed. Different kinds of sensors can be used to capture the surrounding traffic context, such as the camera, radar, and Lidar systems [48]-[51]. Bernt et al. designed a Hidden Markov Model (HMM) based intention classifier which takes the distance to the next turn, the street curvature, and street type from the digital map as the algorithm inputs [52]. Rafael et al. predicted the lane change maneuvers on highways with GPS/IMU sensors to collect the vehicle position [53]. One of the advantages of the GPS is that it gives the location and time information in unfavorable weather conditions when the camera and radar system cannot work. In [54], McCall and Trivedi proposed a preliminary work that focuses on the lane change events. Radar and video devices are used to obtain the forward, rear, and side information. Meanwhile, cameras were also used to monitor the driver foot gesture and head movement. The work in [55] concentrated on the lane change intention prediction according to the sensory data, which contains the lane information given by a lane tracker, the vehicle velocity, lateral position and its derivation, and the steering wheel angle. 2) Vehicle Dynamics

Vehicle dynamic information such as steering wheel angle, brake pedal position, and velocity is the direct response to the control actions from the driver. Hence, these signals have been widely adopted for driver intention identification in the past. Vehicle data are usually collected from the CAN bus, which enables a large amount of data collection with high transfer speed [50] [52] [56]. Schmidt et al. proposed a lane change intention recognition method based on the construction of an explicit mathematical model of the steering wheel [57]. In [58] [59], driver lane change/keep intention inference systems were proposed on a driving simulator with the collection of vehicle dynamic information. In [60], an intention recognition method with artificial neural networks (ANN) was proposed. CAN bus data and driver gaze information was collected and fed into the ANN. However, since vehicle dynamic information is the response to the driving actions, they give delayed information compared with driver behavior data and traffic context information in the intention inference tasks. In general, vehicle dynamic information cannot provide advanced information for intention prediction. However, they still useful for the intention identification and can help to recognize the intent at an early stage after the intended maneuver has been initiated.

\section{3) Driver Behaviors}

Unlike the vehicle dynamic data, driver behavioral signals such as the head and eye movement give an early clue about the driver intention. Many studies have evaluated the impact of head/eye movement on the intention prediction [61]-[66]. Typically driver eye movement can be classified into intention-oriented and non-intention-oriented. Intention oriented eye movement means that the eye fixation or saccades is in purpose, while non-intention-based eye movement is cause by surrounding distractions. Driver visual fixation will no 
longer follow the attention when the driver is distracted. In this case, eye movement can neither reflect driver mental purpose nor the predicted intention being trusted [63]. Regarding the intention-oriented eye tracking, it can be viewed as a cognitive progress of information gathering, which provides an early indication of driver mental states. Besides, the driver intention at the information gathering stage is less likely to change compared than that at the action execution stage [64].

Although head/eye movement can be caused by distraction, most of the time, the driver will shift the eye gaze in purpose, which makes the eye movement an important signal for the intention decoding and inference [65]-[67]. It has been proved that the eye movement information improves the intention prediction accuracy and help to decrease the false alarm rate [68] [69]. A significant challenge to the eye information gathering is the eye tracking task. Dues to the physical characteristics of the eye (small scale and occlusion, etc.), it is not easy to detect the eye and track the pupil robustly. Moreover, the glass, lightness, and even hairs can influence eye tracking performance. According to these challenges, some robust algorithms for eye movement detection have been proposed [70]-[72]. Lethaus et al. [73] evaluated how early and how much data can be used to predict driver intention on a driving simulator. They concluded that a ten seconds window of the eye gaze data is large enough for intention prediction, and a five seconds window gives a better performance since less noise was carried.

Similarly, head motion also reflects the cognitive process of information gathering. It was regarded as the most critical factor for intention prediction [68]. Head movement was widely adopted in DII systems [74] [78]. In [68], the authors claimed that both eye and head movement are useful data for the detection of driver distraction, attention, and mental state inference. However, head moves earlier than the eye when the driver is executing a goal-oriented task. On the other hand, when an outside stimulus occurs, and the driver is facing a non-goal-oriented task, she/he will shift her/his eye before rotating the head [68]. This is an interesting conclusion since it offers a way to determine whether the ongoing driver behavior is goal-oriented or stimuli-based. In [50], the authors evaluated the impact of LDW, ACC, SWA, and head tracking on intention detection. It was found that head tracking is most relevant to the intention recognition and the ACC and SWA systems have limited influence on the lane change intention prediction task.

In addition to the eye gaze and head movement, some other behavioral signals like EEG, foot gesture, hand, and body gesture were also involved in literature [79]-[82]. EEG is an essential sensor for BCI design. EEG is sensitive to the small changes in the electrical activities, which is suitable to detect human mental state. Since EEG measures the brain activities, it can reflect the intention faster than the human muscle reaction. It was found that by using EEG, the braking intention can be detected 130 milliseconds faster than that only consider the brake pedal position [85] [86]. The drawbacks of EEG are the large signal noise, hard to acquire the signal, and getting weak if sampled with poor quality [83]. This is because brain electric current is under the brain layers, skull, and scalp and detected with the non-invasive method [84]. Despite the less robustness in the real-world application, EEG devices are widely accepted on the driving simulators and laboratory environment.

\section{Algorithms for Driver Intention Inference}

In [50], the proposed intelligent vehicle carries more than 200 kinds of sensory signals from the LDW, ACC, SWA, and head tracking system. At this moment, machine learning algorithms are becoming the most suitable tool for data fusion and model construction. As discussed in [32], discriminative models lead to a better result on the single target detection than the generative models, while the generative models are more suitable for the multi-target problems. Despite these two typical methods, driver intention can also be modeled based on the cognitive models and the deep learning models. A taxonomy of the algorithms for intention inference is shown in Fig. 5. In Table 2, the comparison between different LCII systems are illustrated. In Table 2, signals and algorithm represent the model inputs and algorithms used to construct the inference model. The on-road environment means the data are collected in real-world while simulator means the experiment does not have naturalist on-road data and all the data are collected with a driving simulator. The number of subject measures how many subjects or how many data are involved in the experiment. The performance and prediction horizon are two different evaluation metrics that will be further discussed in the next part.

\section{1) Generative Model}

Generative models like HMM are widely used in existing LCII studies [52] [57]-[62] [87] [88]. In [69], the authors used three different algorithms, which were ANN, Bayesian network $(\mathrm{BN})$, and Naive Bayesian. In [89], a new feature named comprehensive decision index (CDI) was introduced. Fuzzy logic was applied to represent the surrounding environment and driver lane change willingness. Li. et al. proposed an integrated intention inference algorithm based on HMM and Bayesian Filtering (BF) technique [90]. A preliminary output from the HMM was further filtered using the BF method to make the final decision. The HMM-BF framework achieved $93.5 \%$ and $90.3 \%$ recognition accuracy for the right and left lane change, respectively. In [58], the authors proposed a driver lane change/keep intention inference method based on a dynamic Bayesian network (DBN). A four-step framework for the DII was developed, and the auto-regression (AR) was combined with the HMM to take the previous driver behaviors into consideration.

In [91], the classification performance did not show a significant increase with additional traffic context information. However, the authors showed that the additional context information leads to a high false positive and the system performance was worse than the system with vehicle state information only. One possible explanation is that the HMM has limited ability to capture the context information during the lane change process. Therefore, a more powerful algorithm such as double layered HMM and input-output HMM should be used [98]. In [92], a lane change detection method based on the object-oriented $\mathrm{BN}$ was proposed. The system was designed according to the modularity and reusability of the BN, which makes the system easier to be extended according to different requirements. 


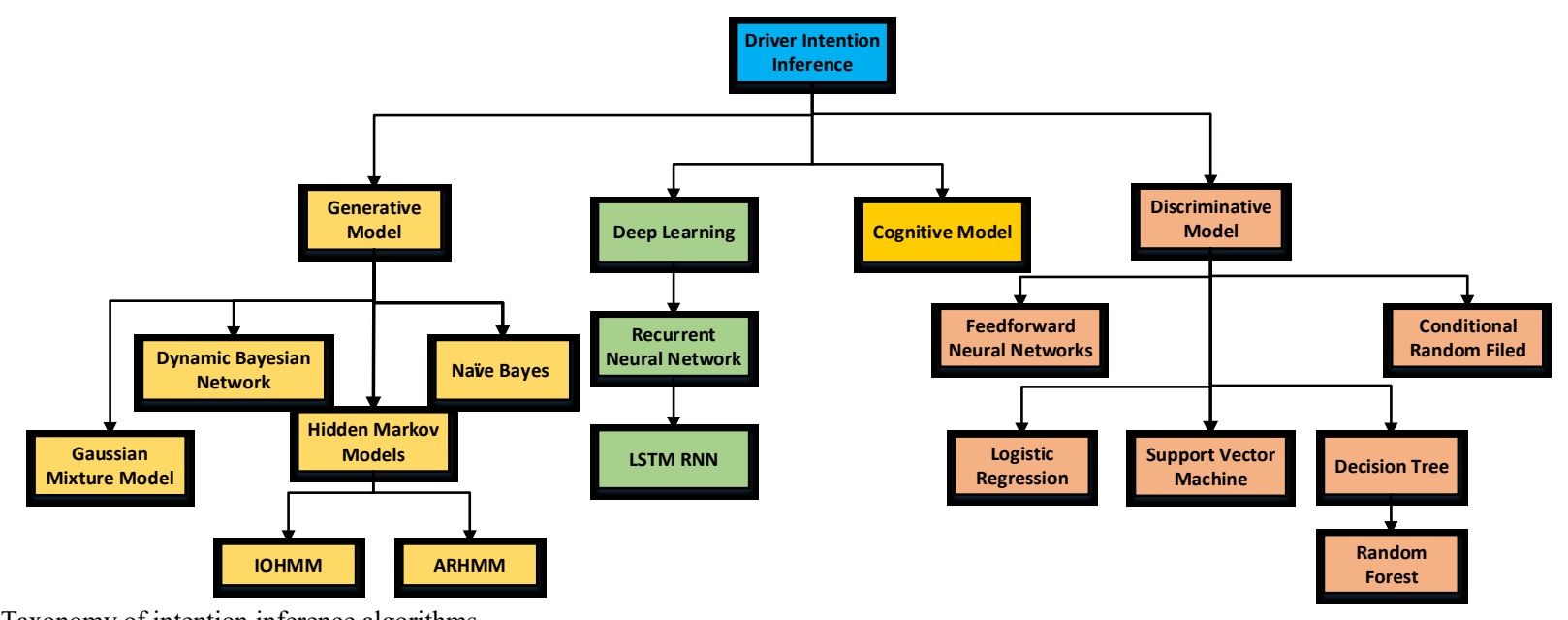

Fig. 5. Taxonomy of intention inference algorithms

TABLE 2

SUMMARIZE OF VARIOUS PREVIOUS LANE CHANGE INTENTION INFERENCE SYSTEMS

\begin{tabular}{|c|c|c|c|c|c|c|}
\hline Paper & Signals & Algorithm & No. Subjects & Environment & Performance & Predict Horizon \\
\hline$[59]$ & $\begin{array}{l}\text { Steering angle, steering } \\
\text { force, velocity }\end{array}$ & CHMM & 10 & Simulator & $100 \%$ & $\begin{array}{l}0.5-0.7 \mathrm{~s} \text { after } \\
\text { steering }\end{array}$ \\
\hline [68] & $\begin{array}{c}\text { Lane position, CAN bus, } \\
\text { Eye and Head }\end{array}$ & RVM & 8 & On-road & $88.51 \%$ & $\begin{array}{l}3 \mathrm{~s} \text { before the lane } \\
\text { change }\end{array}$ \\
\hline [103] & Lane, CAN, and Head & Sparse Bayesian Learning & 3 & On-road & $90 \%$ & $3 \mathrm{~s}$ before maneuver \\
\hline [67] & Eye movement & Finish Questionnaire & 17 & Simulator & $77 \%$ & - \\
\hline [104] & Eye movement & SVM & 24 Samples & On-road & $73.13 \% \pm .25 \%$ & - \\
\hline [52] & CAN, Digital map & HMM & $50 \mathrm{LCL}, 50 \mathrm{LCR}$ & On-road & $71 \% \mathrm{~L}, 74 \% \mathrm{R}$ & - \\
\hline [91] & CAN, Distance & HMM & 20 & On-road & $80 \%-90 \%$ & - \\
\hline [51] & CAN, LDW, ACC, Head, & RVM & 15 & On-road & $91 \%$ & $1 \mathrm{~s}$ prior to maneuver \\
\hline [44] & CAN, Head, Eye & HMM & 70 & On-road & $12.5 \% \mathrm{LR}, 17.6 \mathrm{LL}$ & $\begin{array}{c}\text { 1s prior the } \\
\text { maneuver }\end{array}$ \\
\hline [41] & $\begin{array}{l}\text { CAN, Lane style and } \\
\text { position, Head, Eye }\end{array}$ & Relevance vector machine & 108 Lane changes & On-road & $79.20 \%$ & - \\
\hline [87] & Steering angle & Queuing network model & 14 & Simulator & $\begin{array}{c}\text { LCN } 98.61 \% \text { LCE } \\
91.67 \%,\end{array}$ & - \\
\hline [105] & CAN, Eye movement & State Transition Diagram & $\begin{array}{l}20 \text { (8576 lane } \\
\text { changes) }\end{array}$ & Simulator & $80 \%$ & - \\
\hline$[50]$ & $\begin{array}{l}\text { CAN, ACC, SWA, LDW, } \\
\text { Head }\end{array}$ & RVM & 15 (500 samples) & On-road & $80 \%$ & $\begin{array}{l}3 \mathrm{~s} \text { before the lane } \\
\text { change }\end{array}$ \\
\hline [106] & CAN, GPS, Eye & Finish Questionnaire & 22 & On-road & - & - \\
\hline$[55]$ & $\begin{array}{l}\text { Steering angle and relative } \\
\text { lane position }\end{array}$ & $\begin{array}{l}\text { SVM and Bayesian } \\
\text { Filtering }\end{array}$ & 2 (139 Samples) & On-road & $80 \%$ & $\begin{array}{c}1.3 \mathrm{~s} \text { before the lane } \\
\text { change }\end{array}$ \\
\hline [60] & CAN, Eye & ANN & 10 & Simulator & $95 \%(\mathrm{~L}), 85 \%(\mathrm{R})$ & - \\
\hline [107] & $\begin{array}{c}\text { CAN, Lidar, Radar, Hand, } \\
\text { Head, Foot }\end{array}$ & $\begin{array}{c}\text { Latent Dynamic } \\
\text { Conditional Random Field } \\
\text { (LDCRF) }\end{array}$ & 1000 samples & On-road & $90 \%$ & $\begin{array}{l}\text { 2s prior the lane } \\
\text { change }\end{array}$ \\
\hline [108] & CAN & $\begin{array}{l}\text { SVM and Bayesian } \\
\text { Network }\end{array}$ & 4 & Simulator & $\begin{array}{l}95 \%(\mathrm{LK}) \\
80 \%(\mathrm{LC})\end{array}$ & - \\
\hline [34] & CAN, eye movement & $\begin{array}{c}\text { Computational model } \\
\text { based on ACT-R }\end{array}$ & 11 & Simulator & $90 \%$ & $1 \mathrm{~s}$ after steering \\
\hline [90] & CAN bus & $\begin{array}{l}\text { CHMM and Bayesian } \\
\text { Filtering }\end{array}$ & $\begin{array}{l}\text { 188LCL, } \\
\text { 212LCR, 242 LK }\end{array}$ & On-road & $\begin{array}{l}93.5 \%(\mathrm{~L}) \\
90.3 \%(\mathrm{R})\end{array}$ & $\begin{array}{l}0.5-0.7 \mathrm{~s} \text { after } \\
\text { steering }\end{array}$ \\
\hline [98] & $\begin{array}{l}\text { GPS, digital map, head, } \\
\text { CAN bus }\end{array}$ & LSTM-RNN & $\begin{array}{l}\text { Ten drivers, } 1180 \\
\text { miles }\end{array}$ & On-road & $90.5 \%$ & $\begin{array}{l}3.5 \mathrm{~s} \text { prior the lane } \\
\text { change }\end{array}$ \\
\hline
\end{tabular}

\section{2) Discriminative Model}

Discriminative models such as SVM and ANN are widely used in the past dues to the rich background theories and the successful application experiences [50]-[55], [88]-[94]. In [50] [51], a Bayesian extension to the support vector machine algorithm, namely, the relevance vector machine (RVM) was used to classify the driver lane change (right and left), and lane keeping intention. The classifier achieved $80 \%$ accuracy with a relatively low false alarm rate. The authors in [60] proposed a driver intention recognition method based on artificial neural networks. The detection accuracy for left lane change achieved better detection accuracy than the right lane change. The results indicated that the head rotation had consistent gains between $1.5 \mathrm{~s}$ to $2.5 \mathrm{~s}$ before the lane change maneuver. In [55], a multiclass classifier was constructed by combining the SVM and BF. Results showed that the proposed algorithm can realize an average of $1.3 \mathrm{~s}$ prediction in advance and can achieve a 
maximum prediction horizon of $3.29 \mathrm{~s}$. It was concluded that one of the crucial tasks for intention inference is to improve the performance of the lane tracking system and reduce the false alarm rate.

\section{3) Deep Learning Methods}

Recently, tremendous achievements have been made in the deep learning area dues to the development of deep learning theories, parallel computation hardware, and large-scale annotated datasets, etc. The deep Convolutional Neural Networks (CNN) have achieved state-of-art performance on many computer vision tasks, such as the image classification, segmentation, and object detection domains [95] [97]. Meanwhile, the Recurrent Neural Network (RNN) has achieved significant performance on time-series problems such as natural language processing and image captioning [75] [98]. RNN can be used to process the temporal dependence between the dataset as it allows the weighted connection between previous hidden-layers and the current layer. A long short-term memory (LSTM) scheme was proposed to increase the long-term dependency property and overcome the gradient descent [99]. As aforementioned, DII usually need to take previous driver behaviors and traffic context into consideration. The conventional HMM method has limited ability to capture long-term dependency. While the RNN can provide a better prediction of the driver intention. In [100], an LSTM-RNN model was developed to infer the driver intention when the vehicle enters an interaction. The RNN outperforms the quadratic discriminate analysis model. Similar, a series of studies have been proposed in [98]. The authors compared the LCII performance of the LSTM-based RNN with multiple HMMs. The lane change intent can be detected 3.5 seconds earlier before the vehicle come into another lane. The LSTM-RNN achieved one of the state-of-art results with the precision and recall of $90.5 \%$ and $87.4 \%$.

\section{4) Cognitive Model}

Despite the machine learning algorithms, human cognitive models were also adopted in the past. Salvucci et al. introduced a real-time LCII system based on mind tracking architecture [33] [34]. The mind tracking computational model continuously infers the driver's unobserved intention from the observed actions, which was built based on the Adaptive Control of Thought-Rational (ACT-R) framework. The system achieved $85 \%$ accuracy and $4 \%$ false alarm rate for the lane change intention detection. In [87], the authors constructed a queuing network cognitive architecture to model driver behavior during normal and emergency lane change. The differences between the outputs of the model and the measured data were compared. The proposed method achieved a high accuracy (above 90\%) and low false alarm rate (29.4\%). Comparing with the inference methods based on the eye gaze and head moment, this method can be easily extended into real-world application. However, since the algorithm was based on the steering wheel angle only, it cannot infer the driver intention before the maneuver happens or at a very early stage.

\section{Evaluation of Driver Intention Inference System}

Evaluating the performance of the DII system is essential and lead to a clear understanding of how the system works in the real world. DII system can be evaluated from two aspects, which are the detection accuracy and prediction horizon.

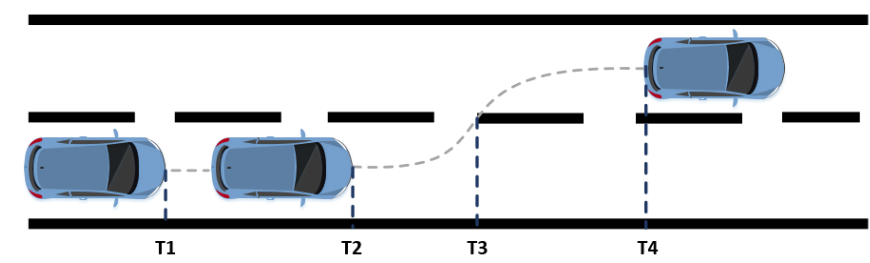

Fig. 6. Illustration of a typical lane change progress with critical moments.

\section{1) Detection Accuracy}

In [94], To evaluate the classification performance, four evaluation criteria were introduced, which were the mean value of prediction horizon, the number of the correctly recognized lane change, the number of not recognizing the lane change, and the number of false alarms. Among these, the true positive rate (TPR) and false positive rate (FPR) are two critical factors to indicate the performance of the classifier. TPR measures how many times the classifier detects the intent successfully, while the FPR describes how many times the classifier miss-classify the intention into the wrong category. Sometimes, FPR is more critical than the TPR since the driver does not want to be disrupted by the assistance system frequently. If a classifier pursues a TPR at the price of high FPR, this system can be hardly accepted by the users. However, if a system has a slightly lower TPR and a lower FPR, it is still helpful in some situations. Therefore, the primary objective is to increase the TPR and decrease the FPR as much as possible [101].

\section{2) Prediction Horizon}

Prediction horizon is another critical factor. Some of the studies reported the TPR and FPR without giving a clear prediction horizon, which was unfair. As shown in Fig. 6, there are four critical moments for a lane change process. T1 represents the moment when the driver generates the lane change intention. $\mathrm{T} 2$ is when the driver finishes traffic context checking and begin to change the lane. T3 represents the moment that the vehicle starts to cross the lane. Finally, the driver completes the lane change task at T4. Because there is no precise driver mental model can be used to explain when exactly the driver generates an intention, $\mathrm{T} 1$ is hard to be precisely determined. Hence, most of the studies use T2 and T3 as the time criteria to evaluate the prediction horizon. The earlier the prediction is made, the more difficult the task will be.

After the driver has taken some actions such as steering the wheel and accelerate/brake, it is straightforward to recognize their intent. However, if the intelligent vehicles try to recognize the driver's intent before the actions are taken, the task will be much more difficult since only limited and uncertain information can be used. As shown in Table 2, some of the studies achieved $90 \%$ prediction accuracy with $2-3$ s prediction horizon [98] [103] [107]. It was reported that the lane change intention can be recognized with a high accuracy $(100 \%$ and 93.5\%) in [59] [90]. However, these results are made after the lane change maneuver has been initiated. The earlier the prediction is made, the higher FPR will be. Therefore, a trade-off between the FPR and the prediction horizon exists, which need to be carefully evaluated [51]. It was found that the data collected $3 \mathrm{~s}$ before the maneuver was enough to present the lane change intention. The prediction horizon in the simulation environment is usually better than that in real-world testing. This is mainly due to the large noise and distraction 
exist in the real-world environment. However, the real-world results indicate a more natural performance, and benefit the analysis of driver mental-physical collaboration

\section{Challenges AND Future Works}

In this section, challenges and part of the future works are highlighted. Four primary works to enhance the DII system are discussed, which are the design of next-generation ADAS, driver situation awareness and interaction aware modeling, autonomous driving, and parallel DII, respectively.

\section{A. Design Next-Generation ADAS}

Next-generation ADAS require further advances in driver understanding from outer behaviors, mental status, and sophisticated environment perception. As aforementioned, current ADAS are only equipped with isolated driver status recognition system, which fails to exploit the relationship between different functions. Meanwhile, a holistic traffic context perception system is required according to the fast development of sensors and an onboard computing device. These concerns give rise to the following discussion.

\section{1) Integration of Driver Monitoring Systems}

The studies of driver behavior-oriented assistance systems can be partially summarized into the following aspects, driver attention, driver intention, driver workload, driver style, and driver distraction, etc. For each research area, a vast amount of studies have been proposed. However, there are still no explicit connections between these systems. It is believed that driver behavior under the distracted condition, and the non-distracted state is different [109] [110]. Also, if the driver is overloaded after a long drive, the physical behaviors are also different [111]. In terms of the DII system, how to correctly infer driver intention with different mental status need to be studied. Therefore, the construction of a robust DII, which can adapt to different driver status is expected. Also, by considering driver monitoring systems has a whole, the control conflicts between the driver and the vehicle can be reduced.

\section{2) The Need for Comprehensive Environment Model.}

Sensing efficiently and precisely is another emerging requirement for the context perception module. A holistic approach is needed in the future to construct a comprehensive environment model from both sensors' view as well as the drivers' view. The driver-oriented context perception must process the context data sequence and analysis the potential driving solutions for the human driver. This can be treated as active guidance that can influence the driver intention generation process rather than only provide the fused context data to the driver and infer the intention afterward. Dynamic analysis of the potential driving behaviors concerning the current context will significantly increase the intention prediction horizon and accuracy. However, real-time estimation leads to a more stringent requirement to onboard perception and computing hardware.

\section{3) Design Cognitive Model for Driver Intention}

A more challenge work is to exploit a comprehensive understanding of the intention generation process according to the traffic context and human behaviors. Currently, driver attention and workload can be mathematically modeled, which provide a better explanation for the driver cognitive attention and workload behaviors [113] [114]. However, there are still limited studies on the explicit modeling of driver intention. Describing the intention generation process with more precise cognitive language and a mathematical model would be one of the core studies in the future.

\section{B. Situation Awareness and Interaction Aware}

The prediction of driver maneuver and the vehicle trajectory needs to be made according to the driver situation awareness and interaction behaviors. In [115], three kinds of vehicle motion modeling methods were proposed, which were the physics-based motion model, maneuver-based motion model, and interaction-aware motion model. The maneuver-based motion model predicts the vehicle trajectory based on the early recognition of the driver intended maneuvers, which is like the intention inference task described in this study. However, most of the maneuver-based models assume the surrounding vehicles move independently without interacting with each other, which can be unreasonable in some complicated situations such as in the roundabout or urban area. Therefore, the interaction-aware modeling methods with respect to the driver situation awareness should be further studied in the future. This part will discuss this problem from two points, which are driver situation awareness modeling and interaction-awareness modeling.

\section{1) Situation Awareness Modeling}

Driver situation awareness (SA) can be viewed as the knowledge that learned and updated from the driving tasks to handle the multifaced situation and guide the driver to make decisions when engaged in real-time multitasking [116]. The perceptual and cognitive process of maintaining the SA also can be divided into three categories, which are automatic (usually unconscious and require no cognitive resources), recognition-primed process (few demands on cognitive resources), and conscious controlled process which requires heavy cognitive resources [116]. Driver SA model carries the habit, knowledge, and attitude towards the specific driving tasks and closely related to the DII since the SA knowledge direct how to understand the driver correctly. For example, a driver intention-oriented situation awareness system at the intersection has been discussed in [27]. Four significant contributions of the situation awareness system are summarized as avoidance of unnecessary warnings, detection of occluded traffic participants, enhancement of driver intent inference, and helps to predict future trajectories of other entities.

Regarding the lane change maneuvers, the four factors are also important since situation awareness model enables the analysis of surrounding traffic flow and provide guidance to the DII system. In [117], driver lane change maneuver was classified into five categories based on the different interaction style with surrounding vehicles. With the analysis of 1000 naturalistic highway lane change data, it was found that $72 \%$ of the lane change was self-motivated and had no significant interaction with the surrounding vehicles. However, without the proper SA, drivers may be unable to finish the intended maneuver smoothly when encountering a complex interaction. For example, a low-speed vehicle is in front of the ego-lane and a rear vehicle is fast approaching in the overtaking lane. In this case, the driver may wish to overtake the front vehicle and must control the vehicle according to the SA and the motion prediction of the rear vehicle. If the driver may postpone his 
lane change maneuver and let rear vehicle pass first, a conflict will be generated between the desired intention and the actual maneuver.

Most of the driver intention studies in the past do not provide enough analysis of this conflict because the driver intention is unable to be predicted and labeled precisely, especially in such a complex condition. Further, as mentioned in [117], most of the naturalistic lane change maneuvers have no significant interaction with other vehicles. The complex interaction scenarios are hard to be repeated in the real world so that not enough data can be used to analyze the conflict situations. However, the situation assessment and understanding can be used to predict the dangerous maneuver at the intersections so that conflict between the actual intention and the expected intention can be clarified [118]. Specifically, the intended stop/go maneuver and the expected maneuver of the driver when approaching an intersection was compared to gain a risk assessment of the dangerous maneuver. In [119], the context information and the corresponding traffic rules were applied with the DBN so that the expected maneuver of the driver can be estimated. The future motion of the traffic participants is the combination of the tactical intentions and their corresponding risk assessment to perform the maneuver [120]. Therefore, it is believed that driver situation awareness and risk assessment can contribute to a better prediction of driver intention.

In sum, traffic situation awareness concerning the assessment of the traffic contexts, traffic rules, road layout, and driver behaviors, etc. are critical to the correct prediction to the driver intention. A driver may generate a series of checking behaviors and perform the maneuver after the intention. However, the intended maneuver may be postponed or aborted dues to the inappropriate situation. Hence, a comprehensive situation awareness model is needed to fully understand the driver behavior, cognitive process, perception, and interaction habit so that a precise prediction of the driver intention can be achieved.

\section{2) Interaction-Aware Modeling}

The interaction-aware motion prediction assumed traffic entities influence each other and provide a longer-term motion prediction of other road users as the mutual dependencies between the drivers' decisions are considered. Regarding the lane change maneuver, a suddenly cut-in maneuver in front of the ego-vehicle can cause a lane change decision to the ego-driver to avoid collision [121]. At this moment, the DII algorithms may become less powerful than with the interaction-aware algorithms in the prevention of collision as DII is mainly designed for the prediction of active intention. Here we roughly define the active intention as a goal-oriented intention while the passive intention is mainly caused by other road entities and the host driver must finish a specific maneuver in a short period. In [122], an integrated interaction-aware motion prediction model was proposed based on the combination of model-based intention estimation for surrounding entities and learning-based lateral motion prediction. The proposed method provides a reliable estimation of the future planning of the surrounding vehicles and the average prediction time before the lane change maneuvers can be extended by more than $60 \%$.

In [123], a unified framework for maneuver classification, trajectory prediction, and interaction-aware motion prediction was proposed. It was shown that the predicted surrounding vehicle motion should be determined according to the comprehensive analysis of the potential maneuver and the probability of the future trajectory. In [124], a generic probabilistic interactive situation aware model is proposed based on a two-layer HMM framework (TLHMM). The TLHMM modeled the real-world interaction behaviors in the highway entrance, roundabout, and T-intersections by computing the joint maneuver distribution of the multiple interactive agents. However, the model has a limitation in the long-term prediction since the TLHMM cannot precisely remember the long-term dependency and temporal patterns. With interaction-aware prediction model, the long-term motion and intention of surrounding entities can be estimated and used for host driver intention inference. This will lead to a holistic understanding of current traffic context and enhance the DII system with an even earlier prediction. Moreover, the interaction-aware motion prediction enables the inference of suddenly lane change intention (passive intention) as discussed in the cut-in scenario. However, one of the disadvantages of the interaction-aware model is the computational complexity grows exponentially with the increasing number of vehicles [122]. The interaction-aware method relies on a comprehensive perception of the local traffic context, which increases the overall system cost.

Another interesting point is to predict the driver intention and interaction behaviors based on transfer learning. In [124], the second layer of the TLHMM was trained with virtual data and high-level meta-features instead of traffic context information, which can be quickly applied to the real-world target. The complex interaction behaviors and scenarios are hard to be recorded and duplicated in the real-world while it can be carefully designed and sufficiently tested in the simulation environment. Hence, if the knowledge learned from the simulation can be properly transferred into the real-world scenario, the real-world interaction-aware model can be more precise and robust. This is also a major concern of parallel driving and parallel driver, which will be discussed later.

Despite sensing traffic context with onboard multi-sensor fusion, the interaction-aware prediction model can also be constructed based on the vehicle-to-vehicle (V2V) techniques [125]. The V2V communication does not rely on high-cost sensors but can provide efficient interactive communication and situation awareness for the local area vehicles. The intention inference for the host driver and surrounding drivers can be detected and shared even earlier with the V2V techniques. The impact of the interaction-aware motion prediction and the V2V technique to the host driver intention inference have not been adequately studied in the past. Future works are expected in this area so that a risk-free and highly interactive traffic framework can be built.

\section{Autonomous Driving}

The automated driving technology was divided into different levels based on the SAE standard J3016. With Level three or higher intelligence, the autonomous vehicle is responsible for the environment perception, decision making, motion planning, and vehicle control. The automotive industry wishes to replace human drivers with autonomous cars so that human mistakes 
can be avoided. However, it does not mean that driver modeling is not needed in the future.

DII systems require a comprehensive understanding of the driving environment as well as the driver behavioral pattern. The process of intention generation and execution reflects the driver SA regarding the traffic context. Current decision-making algorithms for autonomous vehicles are mainly based on optimization, probabilistic models, and reinforcement learning. Neither of the algorithms takes the driver experience into the loop. The autonomous vehicle makes lane change mainly based on the pre-defined rule base or the probabilistic model like Markov chain. These algorithms usually fail to consider the acceptance of the human passenger. The DII system will provide important guidance to the autonomous vehicle so that the autonomous vehicle can learn how human drivers make lane change as well as when and where to execute the lane change. Meanwhile, combining DII with driving styles is also considerable [126]. Different drivers have different driving styles, and the intention inference system cannot work uniformly. For some situation, gentle drivers prefer to wait before changing the lane while aggressive driver likes the challenge tasks. If the autonomous vehicle takes the different intention pattern from different driver styles, the autonomous vehicle can minimize the uncomfortable driving experience for the passengers.

Another emerging topic for DII towards autonomous decision making and motion planning is to estimate when and where the driver is going to drive [127]. Most of the existing driver intention inference algorithms do not pay attention to the intended position. The position should be estimated with the comprehensive environment perception and driver behaviors in the past few seconds. Current intention inference algorithms enable the intention prediction before the maneuver. However, the intended position estimation is still a difficult task. The positioning pattern learned from the driver can be transferred to the autonomous vehicle more straightforward. The path planning model can take the estimated short-term destination into calculation so that a more reasonable and human-like path can be generated. Therefore, transferring the DII knowledge to the autonomous vehicle will bring more naturalistic human-like behaviors in both decision-making and motion planning stage.

\section{Parallel Driver Intention Inference System}

As aforementioned, the driver intention inference system suffers from hardware and algorithm limitation. Also, there is still no explicit model to describe the real mental intention process. One of the emerging challenges is short of data for model training and model evaluation. It is hard to collect plenty of real-world data to increase the data diversity since it dramatically increases the temporal and financial cost. Therefore, a novel approach is required to sufficiently train and evaluate the intention inference system, and it would be better to have a self-learning ability to exploit the unseen pattern and principles that are behind the driver intention nature. Fei-Yue Wang first developed the parallel theory in 2004 [128]. The construction of a parallel system requires the ACP approach as the background knowledge, which is the combination of Artificial society, Computational experiments, and Parallel execution [129].

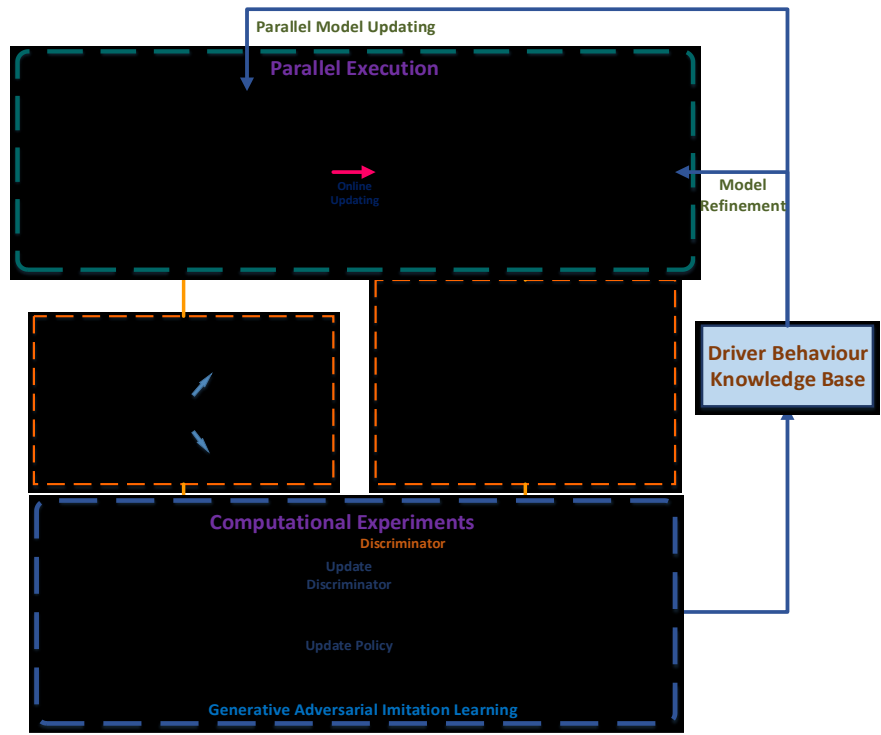

Fig. 7. Architecture of ACP-based parallel driver intention inference system.

The physical system in the real world can be viewed as a Newton machine, whereas the software defined-artificial world is a Merton machine [130]. In [131], the parallel system is described in the Cyber-Physical-Social space (CPSS), which extend the conventional Cyber-Physical space (CPS) by integrating an additional dimension of human and social characteristics. Based on the ACP approach, a parallel driver intention inference system is proposed in Fig. 7.

In the artificial society, a virtual driving environment will be developed based on the modeling of traffic context as well as the driver behaviors such as head, facial, and body features [132]. There are plenty of simulation software that can build the 3D driving context, such as the CarSim or PanoSim. The virtual facial images and videos can be generated based on the high-resolution 3D scans as used in [133]. The driver facial dynamic model can be trained according to the real driver patterns using deep learning approaches such as the generative adversarial networks (GAN) [134]. Then, a generative adversarial imitation learning method can be used to train the virtual driver model [135]. The virtual driver will be sufficiently evaluated with the data from both the artificial world and the real world. Finally, the learned driver behavior knowledge concerning the current traffic context can be used for the training and testing of the driver intention inference model. If the virtual model gives better inference accuracy, it will guide the real-world model to deal with challenge tasks and update the real-world model with online learning methods. With the parallel driver intention inference system, the intention inference model can be trained and evaluated with much more scenarios so that a more robust intention inference model is generated.

\section{CONCLUSION}

Based on this review, driver intention inference is believed an important function for ADAS and intelligent vehicles, which is able to reduce the conflicts between the driver and the intelligent vehicle. Understanding of human intention also enables a better design of the decision-making algorithms for automated vehicles. In this study, the relationship between 
tactical and control level intention is clarified. Based on the DII framework, the traffic context is viewed as the stimuli for the intention, while the driver behavioral information and vehicle dynamics are the response to the stimuli. A comprehensive evaluation method for the intention inference should consider from the aspect of accuracy and prediction horizon. Future works for driver intention inference should concentrate on the precise modeling of the intention generation process, situation and interaction awareness, and autonomous vehicles. Meanwhile, it is believed that a parallel DII framework will dramatically increase the performance of the DII systems.

\section{REFERENCES}

[1] Koesdwiady, Arief, et al. "Recent Trends in Driver Safety Monitoring Systems: State of the Art and Challenges." IEEE Transactions on Vehicular Technology 66.6 (2017): 4550-4563.

[2] Bellis, Elizabeth, and Jim Page. National motor vehicle crash causation survey (NMVCCS) SAS analytical users manual. No. HS-811 053. 2008.

[3] Martinez, Clara Marina, et al. "Driving style recognition for intelligent vehicle control and advanced driver assistance: A survey." IEEE Transactions on Intelligent Transportation Systems (2017).

[4] Michałek, Maciej Marcin, and Marcin Kiełczewski. "The concept of passive control assistance for docking maneuvers with n-trailer vehicles." IEEE/ASME Transactions on Mechatronics 20.5 (2015): 2075-2084.

[5] Wang, Fei-Yue, and Shu-ming Tang. "Concepts and frameworks of artificial transportation systems." Complex Systems and Complexity Science 1.2 (2004): 52-59.

[6] Butakov, Vadim A., and P. Ioannou. "Personalized Driver/Vehicle Lane Change Models for ADAS." IEEE Transactions on Vehicular Technology 64.10(2015):4422-4431.

[7] Son, Young Seop, et al. "Robust multirate control scheme with predictive virtual lanes for lane-keeping system of autonomous highway driving." IEEE Transactions on Vehicular Technology 64.8 (2015): 3378-3391.

[8] He, Jibo, Jason S. McCarley, and Arthur F. Kramer. "Lane keeping under cognitive load: performance changes and mechanisms." Human factors 56.2 (2014): 414-426.

[9] Carruthers, Peter. "The illusion of conscious will." Synthese 159.2 (2007): 197-213.

[10] Ajzen, Icek. "The theory of planned behavior." Organizational behavior and human decision processes 50.2 (1991): 179-211.

[11] Bratman, Michael. "Intention, plans, and practical reason." (1987).

[12] Heinze, Clint. Modelling intention recognition for intelligent agent systems. No. DSTO-RR-0286. DEFENCE SCIENCE AND TECHNOLOGY ORGANISATION SALISBURY (AUSTRALIA) SYSTEMS SCIENCES LAB, 2004

[13] Tahboub, Karim A. "Intelligent human-machine interaction based on dynamic Bayesian networks probabilistic intention recognition." Journal of Intelligent and Robotic Systems 45.1 (2006): 31-52.

[14] Bonchek-Dokow, Elisheva, Cognitive Modeling of Human Intention Recognition. Diss. Bar Ilan University, Gonda Multidisciplinary Brain Research Center, 2011.

[15] Bösch, Holger, Fiona Steinkamp, and Emil Boller . "Examining psychokinesis: The interaction of human intention with random number generators--A meta-analysis." Psychological bulletin 132.4 (2006): 497.

[16] Chadalavada, Ravi Teja, et al. "That's on my mind! robot to human intention communication through on-board projection on shared floor space." Mobile Robots (ECMR), 2015 European Conference on. IEEE, 2015.

[17] Huang, Jian, et al. "Control of upper-limb power-assist exoskeleton using a human-robot interface based on motion intention recognition." IEEE transactions on automation science and engineering 12.4 (2015): $1257-1270$.

[18] Han, Ji-Hyeong, Seung-Jae Lee, and Jong-Hwan Kim. "Behavior Hierarchy-Based Affordance Map for Recognition of Human Intention and Its Application to Human-Robot Interaction." IEEE Transactions on Human-Machine Systems 46.5 (2016): 708-722.

[19] Jang, Young-Min, et al. "Human intention recognition based on eyeball movement pattern and pupil size variation." Neurocomputing 128 (2014): 421-432.
[20] Li, Songpo, and Xiaoli Zhang. "Implicit Intention Communication in Human-Robot Interaction Through Visual Behavior Studies." IEEE Transactions on Human-Machine Systems 47.4 (2017): 437-448.

[21] Takeda, Takahiro, Yasuhisa Hirata, and Kazuhiro Kosuge. "Dance step estimation method based on HMM for dance partner robot." IEEE Transactions on Industrial Electronics 54.2 (2007): 699-706.

[22] Al-Sultan, Saif, A. H. Al-Bayatti, and H. Zedan. "Context-Aware Driver Behavior Detection System in Intelligent Transportation Systems." IEEE Transactions on Vehicular Technology 62.9(2013):4264-4275.

[23] Oliver, Nuria M., Barbara Rosario, and Alex P. Pentland. "A Bayesian computer vision system for modeling human interactions." IEEE transactions on pattern analysis and machine intelligence 22.8 (2000): 831-843.

[24] Bellis, Elizabeth, and Jim Page. National motor vehicle crash causation survey (NMVCCS) SAS analytical users manual. No. HS-811 053. 2008.

[25] Qu, Fengzhong, et al. "A security and privacy review of VANETs." IEEE Transactions on Intelligent Transportation Systems 16.6 (2015): 2985-2996.

[26] Liu, Wei, et al. "Parking like a human: a direct trajectory planning solution." IEEE Transactions on Intelligent Transportation Systems 18.12 (2017): 3388-3397.

[27] Liebner, Martin, and Felix Klanner. "Driver intent inference and risk assessment." Handbook of Driver Assistance Systems: Basic Information, Components and Systems for Active Safety and Comfort (2014): 1-20.

[28] Lv, Chen, et al. "Characterization of Driver Neuromuscular Dynamics for Human-Automation Collaboration Design of Automated Vehicles." IEEE/ASME Transactions on Mechatronics (2018)

[29] Kim, Hyung Jun, and Ji Hyun Yang. "Takeover requests in simulated partially autonomous vehicles considering human factors." IEEE Transactions on Human-Machine Systems 47.5 (2017): 735-740.

[30] Eriksson, Alexander, and Neville A. Stanton. "Takeover time in highly automated vehicles: noncritical transitions to and from manual control." Human factors 59.4 (2017): 689-705.

[31] Michon, John A. "A critical view of driver behavior models: what do we know, what should we do?." Human behavior and traffic safety. Springer, Boston, MA, 1985. 485-524.

[32] Doshi, Anup, and Mohan M. Trivedi. "Tactical driver behavior prediction and intent inference: A review." Intelligent Transportation Systems (ITSC), 2011 14th International IEEE Conference on. IEEE, 2011.

[33] Salvucci, Dario D., and Andrew Liu. "The time course of a lane change: Driver control and eye-movement behavior." Transportation research part F: traffic psychology and behavior 5.2 (2002): 123-132.

[34] Salvucci, Dario D. "Modeling driver behavior in a cognitive architecture." Human factors 48.2 (2006): 362-380.

[35] Lv, Chen, et al. "Hybrid-Learning-Based Classification and Quantitative Inference of Driver Braking Intensity of an Electrified Vehicle. IEEE Transactions on Vehicular Technology. PP.99(2018):1-1.

[36] Haufe, Stefan, et al. "Electrophysiology-based detection of emergency braking intention in real-world driving." Journal of neural engineering 11.5 (2014): 056011.

[37] Khaliliardali, Zahra, et al. "Detection of anticipatory brain potentials during car driving." Engineering in Medicine and Biology Society (EMBC), 2012 Annual International Conference of the IEEE. Ieee, 2012.

[38] McCall, Joel C., and Mohan M. Trivedi. "Driver behavior and situation aware brake assistance for intelligent vehicles." Proceedings of the IEEE 95.2 (2007): 374-387.

[39] Tran, Cuong, Anup Doshi, and Mohan Manubhai Trivedi. "Modeling and prediction of driver behavior by foot gesture analysis." Computer Vision and Image Understanding 116.3 (2012): 435-445.

[40] Ohn-Bar, Eshed, et al. "On surveillance for safety critical events: In-vehicle video networks for predictive driver assistance systems." Computer Vision and Image Understanding 134 (2015): 130-140.

[41] Mabuchi, Ryuki, and Keiichi Yamada. "Study on driver-intent estimation at yellow traffic signal by using driving simulator." Intelligent Vehicles Symposium (IV), 2011 IEEE. IEEE, 2011.

[42] Takahashi, Hiroshi, and Kouichi Kuroda. "A study on mental model for inferring driver's intention." Decision and Control, 1996., Proceedings of the 35th IEEE Conference on. Vol. 2. IEEE, 1996.

[43] Kumagai, Toru, et al. "Prediction of driving behavior through probabilistic inference." Proc. 8th Intl. Conf. Engineering Applications of Neural Networks. 2003.

[44] Oliver, Nuria, and Alex P. Pentland. "Graphical models for driver behavior recognition in a smartcar." Intelligent Vehicles Symposium, 2000. IV 2000. Proceedings of the IEEE. IEEE, 2000. 
[45] Liebner, Martin, et al. "Driver intent inference at urban intersections using the intelligent driver model." Intelligent Vehicles Symposium (IV), 2012 IEEE. IEEE, 2012.

[46] Liu, Andrew, and Alex Pentland. "Towards real-time recognition of driver intentions." Intelligent Transportation System, 1997. ITSC'97., IEEE Conference on. IEEE, 1997.

[47] Imamura, Takashi, et al. "Estimation for driver's intentions in straight road environment using hidden markov models." Systems Man and Cybernetics (SMC), 2010 IEEE International Conference on. IEEE, 2010.

[48] Beauchemin, Steven S., et al. "Portable and scalable vision-based vehicular instrumentation for the analysis of driver intentionality." IEEE Transactions on Instrumentation and Measurement 61.2 (2012): 391-401.

[49] Vahidi, Ardalan, and Azim Eskandarian. "Research advances in intelligent collision avoidance and adaptive cruise control." IEEE transactions on intelligent transportation systems 4.3 (2003): 143-153.

[50] Morris, Brendan, Anup Doshi, and Mohan Trivedi. "Lane change intent prediction for driver assistance: On-road design and evaluation." Intelligent Vehicles Symposium (IV), 2011 IEEE. IEEE, 2011.

[51] Doshi, Anup, Brendan Morris, and Mohan Trivedi. "On-road prediction of driver's intent with multimodal sensory cues." IEEE Pervasive Computing 10.3 (2011): 22-34.

[52] Berndt, Holger, Jorg Emmert, and Klaus Dietmayer. "Continuous driver intention recognition with hidden markov models." Intelligent Transportation Systems, 2008. ITSC 2008. 11th International IEEE Conference on. IEEE, 2008.

[53] Toledo-Moreo, Rafael, and Miguel A. Zamora-Izquierdo. "IMM-based lane-change prediction in highways with low-cost GPS/INS." IEEE Transactions on Intelligent Transportation Systems 10.1 (2009): 180-185.

[54] McCall, Joel C., et al. "A collaborative approach for human-centered driver assistance systems." Intelligent Transportation Systems, 2004. Proceedings. The 7th International IEEE Conference on. IEEE, 2004.

[55] Kumar, Puneet, et al. "Learning-based approach for online lane change intention prediction." Intelligent Vehicles Symposium (IV), 2013 IEEE. IEEE, 2013.

[56] Kumagai, Toru, et al. "Prediction of driving behavior through probabilistic inference." Proc. 8th Intl. Conf. Engineering Applications of Neural Networks. 2003.

[57] Schmidt, Kim, et al. "A mathematical model for predicting lane changes using the steering wheel angle." Journal of safety research 49 (2014): 85-e1.

[58] Li, Fang, et al. "Driving intention inference based on dynamic Bayesian networks." Practical Applications of Intelligent Systems. Springer, Berlin, Heidelberg, 2014. 1109-1119.

[59] Hou, Haijing, et al. "Driver intention recognition method using continuous hidden markov model." International Journal of Computational Intelligence Systems 4.3 (2011): 386-393.

[60] Lethaus, Firas, et al. "Using pattern recognition to predict driver intent." International Conference on Adaptive and Natural Computing Algorithms. Springer, Berlin, Heidelberg, 2011.

[61] Jang, Young-Min, Rammohan Mallipeddi, and Minho Lee. "Identification of human implicit visual search intention based on eye movement and pupillary analysis." User Modeling and User-Adapted Interaction 24.4 (2014): 315-344.

[62] Pech, Timo, Philipp Lindner, and Gerd Wanielik. "Head tracking based glance area estimation for driver behavior modelling during lane change execution." Intelligent Transportation Systems (ITSC), 2014 IEEE 17th International Conference on. IEEE, 2014.

[63] Shinar, David. "Looks are (almost) everything: where drivers look to get information." Human Factors 50.3 (2008): 380-384.

[64] Caceres, N., J. P. Wideberg, and F. G. Benitez. "Deriving origindestination data from a mobile phone network." IET Intelligent Transport Systems 1.1 (2007): 15-26.

[65] Borji, Ali, Andreas Lennartz, and Marc Pomplun. "What do eyes reveal about the mind?: Algorithmic inference of search targets from fixations." Neurocomputing 149 (2015): 788-799.

[66] Doshi, Anup, and Mohan Trivedi. "Investigating the relationships between gaze patterns, dynamic vehicle surround analysis, and driver intentions." Intelligent Vehicles Symposium, 2009 IEEE. IEEE, 2009.

[67] Zhou, Huiping, Makoto Itoh, and Toshiyuki Inagaki. "Toward inference of driver's lane-change intent under cognitive distraction." SICE Annual Conference 2010, Proceedings of. IEEE, 2010.

[68] Doshi, Anup, and Mohan Manubhai Trivedi. "On the roles of eye gaze and head dynamics in predicting driver's intent to change lanes." IEEE Transactions on Intelligent Transportation Systems 10.3 (2009): 453-462.
[69] Lethaus, Firas, et al. "A comparison of selected simple supervised learning algorithms to predict driver intent based on gaze data." Neurocomputing 121 (2013): 108-130.

[70] Timm, Fabian, and Erhardt Barth. "Accurate Eye Centre Localisation by Means of Gradients." Visapp 11 (2011): 125-130.

[71] Wang, Shuo, et al. "Atypical visual saliency in autism spectrum disorder quantified through model-based eye tracking." Neuron 88.3 (2015): 604-616.

[72] Martin, Sujitha, et al. "Dynamics of Driver's Gaze: Explorations in Behavior Modeling and Maneuver Prediction." IEEE Transactions on Intelligent Vehicles (2018).

[73] Lethaus, Firas, et al. "Windows of driver gaze data: how early and how much for robust predictions of driver intent?." International Conference on Adaptive and Natural Computing Algorithms. Springer, Berlin, Heidelberg, 2013.

[74] Ohn-Bar, Eshed, and Mohan Manubhai Trivedi. "Looking at humans in the age of self-driving and highly automated vehicles." IEEE Transactions on Intelligent Vehicles 1.1 (2016): 90-104.

[75] Jain, Ashesh, et al. "Recurrent neural networks for driver activity anticipation via sensory-fusion architecture." Robotics and Automation (ICRA), 2016 IEEE International Conference on. IEEE, 2016.

[76] Murphy-Chutorian, Erik, and Mohan Manubhai Trivedi. "Head pose estimation in computer vision: A survey." IEEE transactions on pattern analysis and machine intelligence 31.4 (2009): 607-626.

[77] Ahlstrom, Christer, et al. "Processing of eye/head-tracking data in large-scale naturalistic driving data sets." IEEE transactions on intelligent transportation systems 13.2 (2012): 553-564.

[78] Tawari, Ashish, Sujitha Martin, and Mohan Manubhai Trivedi. "Continuous head movement estimator for driver assistance: Issues, algorithms, and on-road evaluations." IEEE Transactions on Intelligent Transportation Systems 15.2 (2014): 818-830.

[79] Tran, Cuong, Anup Doshi, and Mohan Manubhai Trivedi. "Modeling and prediction of driver behavior by foot gesture analysis." Computer Vision and Image Understanding 116.3 (2012): 435-445.

[80] Tiwari, Ravikumar K., and S. D. Giripunje. "Design approach for EEG-based human computer interaction driver monitoring system." Int $J$ Latest Trends Eng Technol IJLTET 3.4 (2014): 250-255.

[81] Das, Nikhil, Eshed Ohn-Bar, and Mohan M. Trivedi. "On performance evaluation of driver hand detection algorithms: Challenges, dataset, and metrics." Intelligent Transportation Systems (ITSC), 2015 IEEE 18th International Conference on. IEEE, 2015.

[82] Xing, Yang, et al. "Identification and Analysis of Driver Postures for In-Vehicle Driving Activities and Secondary Tasks Recognition." IEEE Transactions on Computational Social Systems 5.1 (2018): 95-108.

[83] Nicolas-Alonso, Luis Fernando, and Jaime Gomez-Gil. "Brain computer interfaces, a review." Sensors 12.2 (2012): 1211-1279.

[84] Wang, Xiao-Wei, Dan Nie, and Bao-Liang Lu. "Emotional state classification from EEG data using machine learning approach." Neurocomputing 129 (2014): 94-106.

[85] Haufe, Stefan, et al. "EEG potentials predict upcoming emergency brakings during simulated driving." Journal of neural engineering 8.5 (2011): 056001.

[86] Ikenishi, Toshihito, Takayoshi Kamada, and Masao Nagai. "Classification of Driver Steering Intentions Using an Electroencephalogram." Journal of System Design and Dynamics 2.6 (2008): 1274-1283.

[87] $\mathrm{Bi}$, Luzheng, et al. "Detecting driver normal and emergency lane-changing intentions with queuing network-based driver models." International Journal of Human-Computer Interaction 31.2 (2015): 139-145.

[88] Driggs-Campbell, Katherine, and Ruzena Bajcsy. "Identifying modes of intent from driver behaviors in dynamic environments." Intelligent Transportation Systems (ITSC), 2015 IEEE 18th International Conference on. IEEE, 2015.

[89] Ding, Jieyun, et al. "Driver intention recognition method based on comprehensive lane-change environment assessment." Intelligent Vehicles Symposium Proceedings, 2014 IEEE. IEEE, 2014.

[90] Li, Keqiang, et al. "Lane changing intention recognition based on speech recognition models." Transportation research part C: emerging technologies 69 (2016): 497-514.

[91] Polling, D., et al. "Inferring the driver's lane change intention using context-based dynamic Bayesian networks." Systems, Man and Cybernetics, 2005 IEEE International Conference on. Vol. 1. IEEE, 2005. 
[92] Kasper, Dietmar, et al. "Object-oriented Bayesian networks for detection of lane change maneuvers." IEEE Intelligent Transportation Systems Magazine 4.3 (2012): 19-31.

[93] Mandalia, Hiren M., and Mandalia Dario D. Salvucci. "Using support vector machines for lane-change detection." Proceedings of the human factors and ergonomics society annual meeting. Vol. 49. No. 22. Sage CA: Los Angeles, CA: SAGE Publications, 2005.

[94] Dogan, Ueruen, Hannes Edelbrunner, and Ioannis Iossifidis. "Towards a driver model: Preliminary study of lane change behavior." Intelligent Transportation Systems, 2008. ITSC 2008. 11th International IEEE Conference on. IEEE, 2008.

[95] Girshick, Ross. "Fast r-cnn." arXiv preprint arXiv:1504.08083 (2015).

[96] Lv, Yisheng, et al. "Traffic flow prediction with big data: a deep learning approach." IEEE Transactions on Intelligent Transportation Systems 16.2 (2015): 865-873.

[97] Krizhevsky, Alex, Ilya Sutskever, and Geoffrey E. Hinton. "Imagenet classification with deep convolutional neural networks." Advances in neural information processing systems. 2012.

[98] Jain, Ashesh, et al. "Brain4cars: Car that knows before you do via sensory-fusion deep learning architecture." arXiv preprint arXiv:1601.00740 (2016).

[99] Hochreiter, Sepp, and Jügen Schmidhuber. "Long short -term memory." Neural computation 9.8 (1997): 1735-1780.

[100]Zyner, Alex, Stewart Worrall, and Eduardo Nebot. "A Recurrent Neural Network Solution for Predicting Driver Intention at Unsignalized Intersections." IEEE Robotics and Automation Letters (2018).

[101]Fawcett, Tom. "An introduction to ROC analysis." Pattern recognition letters 27.8 (2006): 861-874.

[102] Imamura, Takashi, et al. "Real-time implementation of estimation method for driver's intention on a driving simulator." Systems, Man and Cybernetics (SMC), 2014 IEEE International Conference on. IEEE, 2014.

[103] McCall, Joel C., et al. "Lane change intent analysis using robust operators and sparse bayesian learning." IEEE Transactions on Intelligent Transportation Systems 8.3 (2007): 431-440.

[104]Jang, Young-Min, Rammohan Mallipeddi, and Minho Lee. "Driver's lane-change intent identification based on pupillary variation." Consumer Electronics (ICCE), 2014 IEEE International Conference on. IEEE, 2014.

[105]Zhou, Huiping, Makoto Itoh, and Toshiyuki Inagaki. "Eye movement-based inference of truck driver's intent of changing lanes." SICE Journal of Control, Measurement, and System Integration 2.5 (2009): 291-298.

[106] Henning, Matthias J., et al. "Modelling driver behavior in order to infer the intention to change lanes." Proceedings of European Conference on Human Centred Design for Intelligent Transport Systems. Vol. 113. 2008.

[107]Ohn-Bar, Eshed, et al. "Predicting driver maneuvers by learning holistic features." Intelligent Vehicles Symposium Proceedings, 2014 IEEE. IEEE, 2014.

[108] Xu, Guoqing, Li Liu, and Zhangjun Song. "Driver behavior analysis based on bayesian network and multiple classifiers." Intelligent Computing and Intelligent Systems (ICIS), 2010 IEEE International Conference on. Vol. 3. IEEE, 2010.

[109]Young, Kristie, Michael Regan, and M. Hammer. "Driver distraction: A review of the literature." Distracted driving (2007): 379-405.

[110]Liao, Yuan, et al. "Detection of driver cognitive distraction: A comparison study of stop-controlled intersection and speed-limited highway." IEEE Transactions on Intelligent Transportation Systems 17.6 (2016): 1628-1637.

[111]Healey, Jennifer A., and Rosalind W. Picard. "Detecting stress during real-world driving tasks using physiological sensors." IEEE Transactions on intelligent transportation systems 6.2 (2005): 156-166.

[112]Klauer, Sheila G., et al. "The impact of driver inattention on near-crash/crash risk: An analysis using the 100-car naturalistic driving study data." (2006).

[113]Regan, Michael A., Charlene Hallett, and Craig P. Gordon. "Driver distraction and driver inattention: Definition, relationship and taxonomy." Accident Analysis \& Prevention 43.5 (2011): 1771-1781.

[114] Ma, Yu-Fei, et al. "A generic framework of user attention model and its application in video summarization." IEEE transactions on multimedia 7.5 (2005): 907-919.

[115]Lefè̀re, Stǿhanie, Dizan Vasquez, and Christian Laugier. "A survey on motion prediction and risk assessment for intelligent vehicles." ROBOMECH journal 1.1 (2014): 1.
[116] Gugerty, Leo. "Situation awareness in driving." Handbook for driving simulation in engineering, medicine and psychology 1 (2011).

[117] Yao, Wen, et al. "On-road vehicle trajectory collection and scene-based lane change analysis: Part II." IEEE Transactions on Intelligent Transportation Systems 18.1 (2017): 206-220.

[118]Lefè̀re, Stథ́hanie, Christian Laugier, and Javier Ibar̃zz -Guzmán. "Evaluating risk at road intersections by detecting conflicting intentions." Intelligent Robots and Systems (IROS), 2012 IEEE/RSJ International Conference on. IEEE, 2012.

[119]Lefè̀re, Stǿhanie, Javier Ibar̃ez -Guzmán, and Christian Laugier. "Context-based estimation of driver intent at road intersections." Computational Intelligence in Vehicles and Transportation Systems (CIVTS), 2011 IEEE Symposium on. IEEE, 2011.

[120]Lawitzky, Andreas, et al. "Interactive scene prediction for automotive applications." Intelligent Vehicles Symposium (IV), 2013 IEEE. IEEE, 2013.

[121]Bahram, Mohammad, et al. "A Game-Theoretic Approach to Replanning-Aware Interactive Scene Prediction and Planning." IEEE Trans. Vehicular Technology 65.6 (2016): 3981-3992.

[122]Bahram, Mohammad, et al. "A combined model-and learning-based framework for interaction-aware maneuver prediction." IEEE Transactions on Intelligent Transportation Systems 17.6 (2016): 1538-1550.

[123]Deo, Nachiket, Akshay Rangesh, and Mohan M. Trivedi. "How Would Surround Vehicles Move? A Unified Framework for Maneuver Classification and Motion Prediction." IEEE Transactions on Intelligent Vehicles 3.2 (2018): 129-140.

[124] Li, Jiachen, et al. "Generic probabilistic interactive situation recognition and prediction: From virtual to real." 201821 st International Conference on Intelligent Transportation Systems (ITSC). IEEE, 2018.

[125] Golestan, Keyvan, et al. "Situation awareness within the context of connected cars: A comprehensive review and recent trends." Information Fusion 29 (2016): 68-83.

[126] Martinez, Clara Marina, et al. "Driving Style Recognition for Intelligent Vehicle Control and Advanced Driver Assistance: A Survey." IEEE Transactions on Intelligent Transportation Systems PP.99(2017):1-11.

[127]Hu, Yeping, Wei Zhan, and Masayoshi Tomizuka. "Probabilistic Prediction of Vehicle Semantic Intention and Motion." IEEE Intelligent Vehicles Symposium. 2018.

[128] Wang Fei-Yue. Parallel system methods for management and control of complex systems. Control and Decision, 2004, 19(5): 485-489.

[129] Wang, F. Y. "Artificial societies, computational experiments, and parallel systems: A discussion on computational theory of complex social-economic systems." Complex Systems \& Complexity Science1.4(2004):25-35.

[130]Wang, Fei Yue. "Parallel Control and Management for Intelligent Transportation Systems: Concepts, Architectures, and Applications." IEEE Transactions on Intelligent Transportation Systems 11.3(2010):630-638.

[131]Wang, Fei Yue, et al. "Parallel Driving in CPSS: A Unified Approach for Transport Automation and Vehicle Intelligence." IEEE/CAA Journal of Automatica Sinica 4.4(2017):577-587.

[132]Y. Xing, et al. End-to-End Driving Activities and Secondary Tasks Recognition Using Deep Convolutional Neural Network and Transfer Learning. IEEE Intelligent Vehicles Symposium. 2018.

[133]J. Gu, X. Yang, S. De Mello, and J. Kautz. Dynamic Facial Analysis: From Bayesian Filtering to Recurrent Neural Networks. IEEE Conference on Computer Vision and Pattern Recognition (CVPR), 2017.

[134] Goodfellow, Ian J, et al. "Generative Adversarial Networks." Advances in Neural Information Processing Systems, (2014):2672-2680.

[135]Ho, Jonathan, and Stefano Ermon. "Generative adversarial imitation learning." Advances in Neural Information Processing Systems. 2016. 


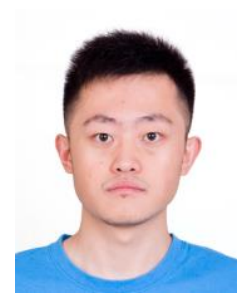

Yang Xing received his $\mathrm{Ph}$. D. degree from Cranfield University, UK in 2018. He is currently a research fellow in the Driver Cognition and Automated Driving $\mathrm{Lab}$ at the University of Waterloo, Canada. His research interests include driver behavior modeling, driver-vehicle interaction, and advanced driver assistance systems. His work focuses on the understanding of driver behaviors and identification of driver intentions using machine-learning methods for intelligent and automated vehicles.

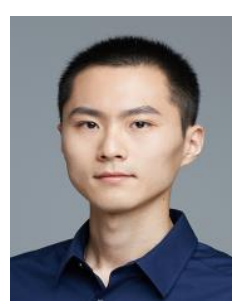

China patents.

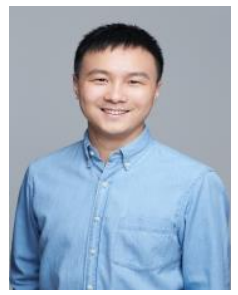

Huaji Wang received the B.S. degree in automotive engineering from Jilin University, China, in 2005, and the $\mathrm{Ph} . \mathrm{D}$. degree in engineering from the University of Cambridge, Cambridge, U.K., in 2016, He is concentrating on the study of driver/vehicle systems and driver-automation collaboration. He presently works as a system engineer in the ALV powertrain, UK.

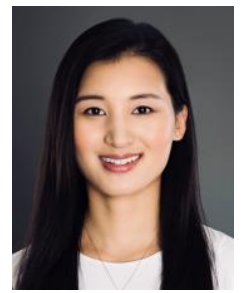

Hong Wang is currently a Research Associate of Mechanical and Mechatronics Engineering with the University of Waterloo. She received her Ph.D. degree in Beijing Institute of Technology in China in 2015. Her research focuses on the path planning control and ethical decision making for autonomous vehicles and component sizing, modeling of hybrid powertrains and power management control strategies design for Hybrid electric vehicles; intelligent control theory and application.

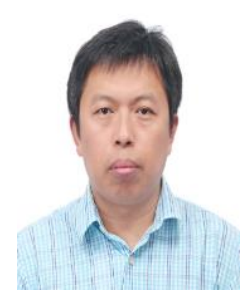

Yunfeng Ai received the B.S. degree from Shandong University, Jinan, China, in 2001 and the Ph.D. degree from the Institute of Automation, Chinese Academy of Sciences, Beijing, China, in 2006. He was a visiting scholar from December 2015 to October 2016 and postdoctoral researcher from November 2016 to April 2017 at Carnegie Mellon University. He is currently a research scientist of the University of Chinese Academy of Sciences. His current research interests include computer vision, machine learning, robots, and

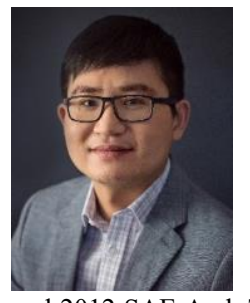

Dongpu Cao received the Ph.D. degree from Concordia University, Canada, in 2008. $\mathrm{He}$ is currently an Associate Professor and Director of Driver Cognition and Automated Driving (DC-Auto) Lab at the University of Waterloo, Canada. His research focuses on vehicle dynamics and control, driver cognition, automated driving and parallel driving, where he has contributed more than 150 publications and 1 US patent. He received the ASME AVTT'2010 Best Paper Award and 2012 SAE Arch T. Colwell Merit Award. Dr. Cao serves as an Associate Editor for IEEE TRANSACTIONS ON VEHICULAR TECHNOLOGY, IEEE TRANSACTIONS ON INTELLIGENT TRANSPORTATION SYSTEMS, IEEE/ASME TRANSACTIONS ON MECHATRONICS, IEEE TRANSACTIONS ON INDUSTRIAL ELECTRONICS and ASME JOURNAL OF DYNAMIC SYSTEMS, MEASUREMENT AND CONTROL. He has been a Guest Editor for VEHICLE SYSTEM DYNAMICS, and IEEE TRANSACTIONS ON SMC: SYSTEMS. He has been serving on the SAE International Vehicle Dynamics Standards Committee and a few ASME, SAE, IEEE technical committees, and serves as Co-Chair of IEEE ITSS Technical Committee on Cooperative Driving.

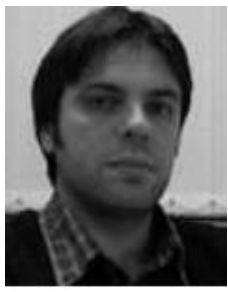

Efstathios Velenis received the Ph.D. degree from the School of Aerospace Engineering, Georgia Institute of Technology, Atlanta, GA, USA, in 2006. He is currently a Senior Lecturer with the Advanced Vehicle Engineering Centre, Cranfield University, Cranfield, U.K. His current research interests include vehicle dynamics and control, optimal control for active chassis systems, traction, braking, and handling control for electric/hybrid vehicles, lap-time optimization, and tire modeling. He obtained research funding from EPSRC, Innovate U.K., and the European Commission. Dr. Velenis received the Luther Long Award for best Ph.D. dissertation in the area of engineering mechanics at Georgia Tech.

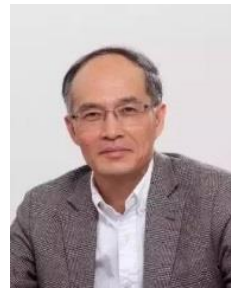

Fei-Yue Wang (S'87-M'89-SM'94-F'03) received his $\mathrm{Ph} . \mathrm{D}$. in Computer and Systems Engineering from Rensselaer Polytechnic Institute, Troy, New York in 1990. He joined the University of Arizona in 1990 and became a Professor and Director of the Robotics and Automation Lab (RAL) and Program in Advanced Research for Complex Systems (PARCS). In 1999, he founded the Intelligent Control and Systems Engineering Center at the Institute of Automation, Chinese Academy of Sciences (CAS), Beijing, China, under the support of the Outstanding Overseas Chinese Talents Program from the State Planning Council and "100Talent Program" from CAS, and in 2002, was appointed as the Director of the Key Lab of Complex Systems and Intelligence Science, CAS. From 2006 to 2010, he was Vice President for Research, Education, and Academic Exchanges at the Institute of Automation, CAS. In 2011, he became the State Specially Appointed Expert and the Director of the State Key Laboratory of Management and Control for Complex Systems. Dr. Wang's current research focuses on methods and applications for parallel systems, social computing, and knowledge automation.

automated driving. 
2019-03-06

\title{
Driver lane change intention inference for intelligent vehicles: framework, survey, and challenges
}

\author{
Xing, Yang
}

IEEE

Yang Xing, Chen Lv, Huaji Wang, et al., Driver lane change intention inference for intelligent vehicles: framework, survey, and challenges. IEEE Transactions on Vehicular Technology, Volume 68, Issue 5, May 2019, pp. 4377-4390

https://doi.org/10.1109/TVT.2019.2903299

Downloaded from Cranfield Library Services E-Repository 